\title{
Slowing of Neurofilament Transport and the Radial Growth of Developing Nerve Fibers ${ }^{1}$
}

\author{
PAUL N. HOFFMAN, ${ }^{\ddagger} \ddagger^{2}$ JOHN W. GRIFFIN,§ BRUCE G. GOLD, ${ }^{\star \star}$ aND DONALD L. PRICE ${ }^{\star} \|$ \\ * Laboratory of Neuropathology, $\ddagger$ The Wilmer Ophthalmological Institute, §Departments of Neurology and Neuroscience, and \\ \|Departments of Neurology, Pathology, and Neuroscience, School of Medicine, and 1 Department of Environmental Health, School of \\ Public Health and Hygiene, The Johns Hopkins University, Baltimore, Maryland 21205
}

\begin{abstract}
Several lines of evidence indicate that neurofilaments are major intrinsic determinants of axonal caliber in myelinated nerve fibers, and that the delivery of neurofilaments by slow axonal transport is an important mechanism by which neurons regulate axonal caliber. To further clarify the relationship between neurofilament transport and axonal caliber, we examined transport in developing motor fibers of rat sciatic nerve. In 3-, 10-, 12-, and 20-week-old rats, lumbar motor neurons were labeled by the intraspinal injection of radioactive amino acids, and the distributions of labeled cytoskeletal proteins within the sciatic nerve were analyzed at various times afterwards using sodium dodecyl sulfate-polyacrylamide gel electrophoresis, gel fluorography, and liquid scintillation spectroscopy. There was a progressive decline in the velocity of neurofilament transport with increasing distance along axons undergoing radial growth. By examining transport in different regions of the nerve in animals of the same age, we separated age-dependent reductions in velocity from those related to position along the nerve. The crosssectional areas of these motor axons (in the $L 5$ ventral root) increased linearly between 3 and 18 weeks of age. Quantitative electron microscopic analysis at 3 and 10 weeks of age revealed that neurofilament density was comparable in fibers of all calibers, indicating that the radial growth of these myelinated nerve fibers correlates with a proportional increase in neurofilament content. We propose that progressive reduction in the velocity of neurofilament transport along the nerve provides for radial growth during development.
\end{abstract}

The caliber of an axon has important functional implications for the neuron in that caliber correlates with the velocity of impulse

Received October 29, 1984; Revised February 19, 1985; Accepted April 10, 1985

'P. N. H. is a John A. and George L. Hartford Foundation Fellow, an Alfred $P$. Sloan Foundation Fellow, and the recipient of Research Career Development Award NII 1 NS 00896. J. W. G. is the recipient of Research Career Development Award NIH NS 00450. This work was supported by United States Public Health Service Grants NIH EY 03791, NS 10580, and NS 15721. We thank Cheryl Auer of the Wilmer Biostatistics Center for carrying out statistical analysis of the data, and Gary Thompson, Kenneth Fahnestock, and Adelaine Stocks for their cxcellent tochnical assistance.

${ }^{2}$ To whom correspondence should be addressed, at 5-167 Meyer Building. The Johns Hopkins University School of Medicine, 600 North Wolfe Street, Baltimore, Maryland 21205. conduction (Hursh, 1939; Minwegen and Friede, 1984), myelin sheath thickness (Friede and Martinez, 1970), internodal distance (within a single nerve fiber), electrical excitability, and, possibly, order of recruitment (Stuart and Enoka, 1983). Although caliber can be influenced by extraneuronal factors (Raine et al., 1969; Weinberg and Spencer, 1975; Aguayo et al., 1979), the major determinants of caliber appear to be intrinsic to the axon (Aguayo et al., 1979). Several lines of evidence indicate that the delivery of neurofilaments by slow axonal transport is an important mechanism by which neurons regulate axonal caliber (Hoffman et al., 1984a, b). To clarify the relationship between neurofilament transport and axonal caliber. we examined transport in developing motor fibers of rat sciatic nerve during a period of sustained radial growth (i.e., between 3 and 18 weeks of age). The results indicate that there is a progressive reduction in the velocity of neurofilament transport with increasing distance along the nerve. This slowing of neurofilament transport may provide a mechanism for radial growth during development.

\section{Materials and Methods}

Labeling axonal proteins in lumbar motor neurons. Male Sprague-Dawley rats, 3 to 20 weeks of age, were used in these studies. Surqical procedures were carried out using chloral hydrate anesthesia $(400 \mathrm{mg} / \mathrm{kg}$, i.p.). Lumbar motor neurons were labeled by the intraspinal administration of microliter quantities of radioactive amino acids according to our published method (Hoffman and Lasek, 1975). In 3-week-old animals, [ $\left.{ }^{35} \mathrm{~S}\right]$ methionine (1200 Ci/ mmol) (Amersham Corp., Arlington Heights, L) was injected at a final concentration of $80 \mathrm{mCi} / \mathrm{ml}$. In 10,12 , and 20 week-old animals, a $1: 1$ mixture of $L-4,5-\left[{ }^{3} \mathrm{H}\right]$ leucine $(30$ to $50 \mathrm{Ci} / \mathrm{mmol})$ and $\mathrm{L}-4,5-\left[{ }^{3} \mathrm{H}\right] \mathrm{lysine}(20$ to 40 $\mathrm{Ci} / \mathrm{mmol}$ ) (both from New England Nuclear Corp., Boston, MA) was injected al a final concentration of $10 \mathrm{mmCi} / \mathrm{rrl}$.

Distribution of labeled proteins in rat sciatic nerve. Animals injected at 10 weeks of age were killed by perfusion with formalin at 33,60, and 85 days after labeling; those injected at 20 weeks were killed 33 days atter labeling. Five animals were analyzed in each group. Sciatic nerves were removed from these animals and divided into 3-mm segments, which were dissolved in Soluene (Packard Instruments, Downers Grove, IL). Levels of radioactivity in the transported proteins (i.e., the entire slow component wave) were measured using liquid scintillation spectroscopy (Hoffman and Lasek, 1975) and plotted as a function of distance along the nerve.

Analysis of labeled proteins using SDS-PAGE fluorography. Animals injected at 3 weeks of age were killed 5, 10, 15, and 20 days after labeling, whereas those injected at 12 weeks were killed at $6,20,33$, and 60 days. Single animals in each age group were analyzed at these postlabeling intervals. To study the distribution of labeled neurofilament protein in the sciatic nerve, we homogenized nerve segments (either 3 or $5 \mathrm{~mm}$ long) in a solution containing $5 \%$ SDS, $8 \mathrm{M}$ urea, and 5\% 2-mercaptoethanol and analyzed the labeled proteins using SDS-PAGE and gel fluorography (Hoffman et al., 1983). Since the 145-kilodalton protein is more completely separated from other labeled gel bands than either the 200- or 68-kilodalton neurofilament proteins, it was used for determining the distribution of neuro- 

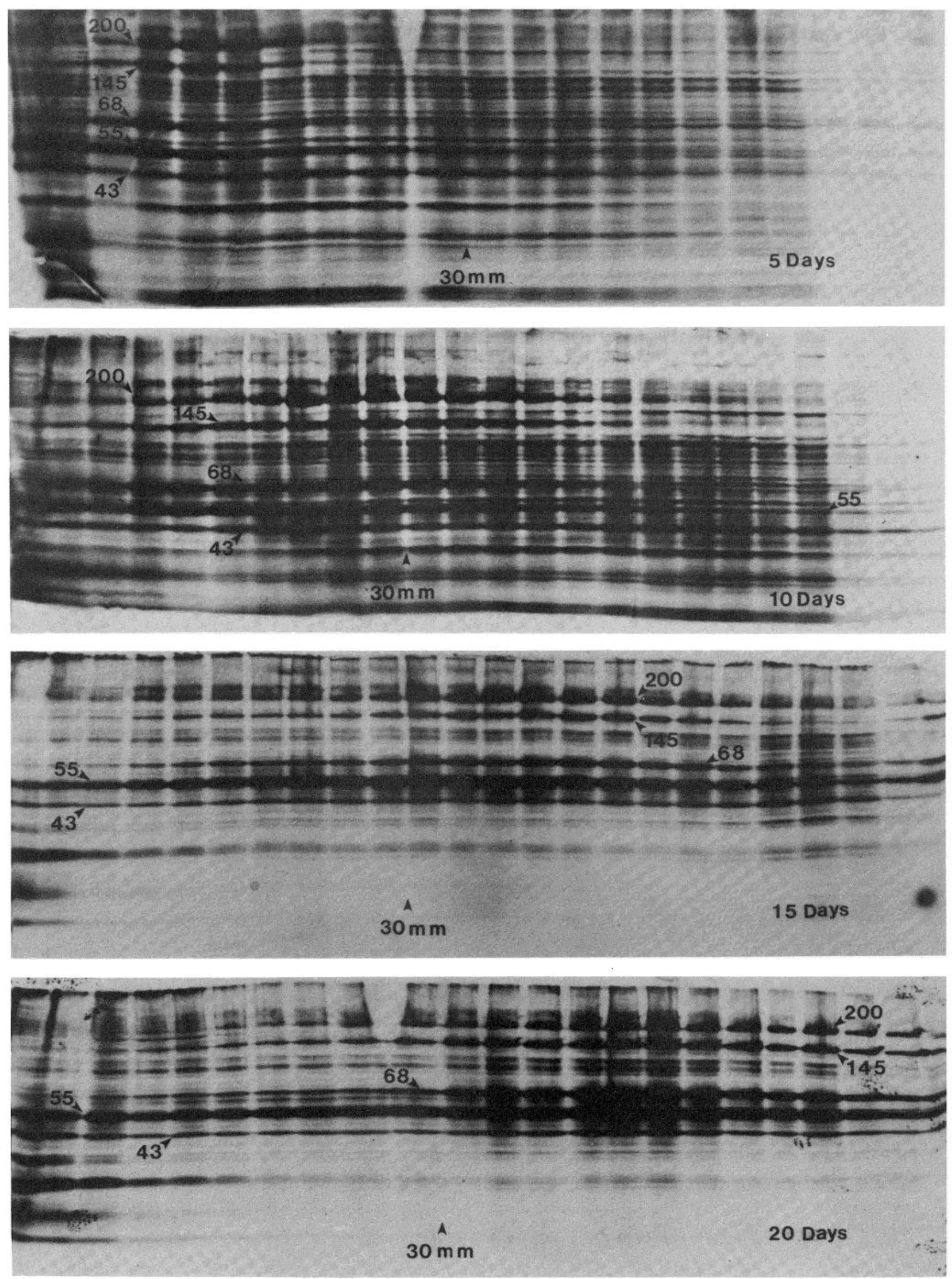

Figure 1. Axonal transport of cytoskeletal proteins in developing motor neurons. These fluorographs of slab gels $(7.5 \%$ acrylamide $)$ illustrate the distribution of the neurofilament proteins $\left(M_{r}=200,000,145,000\right.$, and 68,000), tubulin $\left(M_{r}=55,000\right)$, and actin $\left(M_{r}=43,000\right)$ in the sciatic nerve 5, 10, 15, and 20 days after labeling lumbar motor neurons in 3-week-old animals by the intraspinal injection of [ ${ }^{35}$ S]methionine. Labeled proteins in consecutive 3-mm nerve segments were analyzed in adjacent gel slots. The distributions of these neurofilament proteins coincide at each postlabeling interval. These distributions differ from those of actin and tubulin, which extend more distally at each time interval. In addition, significant amounts of labeled actin and tubulin, but not neurofilament proteins, are retained proximally after passage of the wave containing these proteins. Distance along the nerve (at $30 \mathrm{~mm}$ ) is indicated at the bottom of each gel. Kodak RP $x$-ray film was used, with exposure times ranging from 10 to 25 days. 


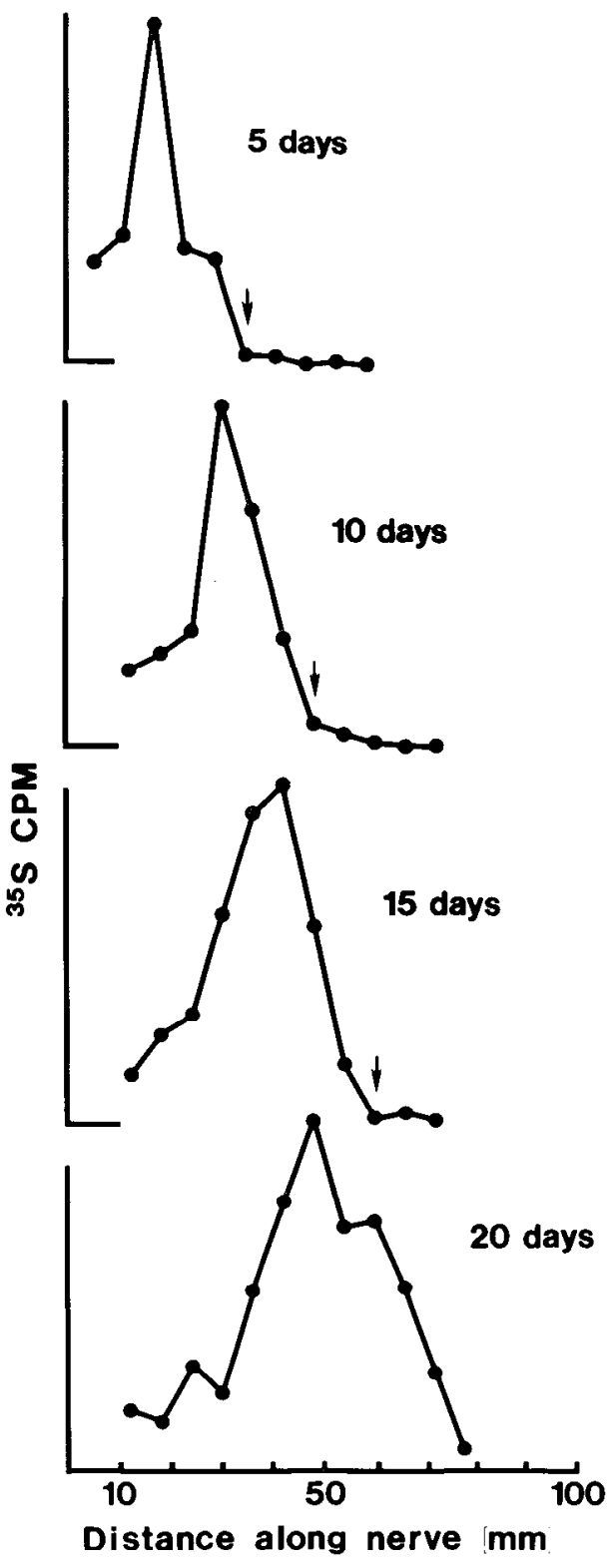

2a

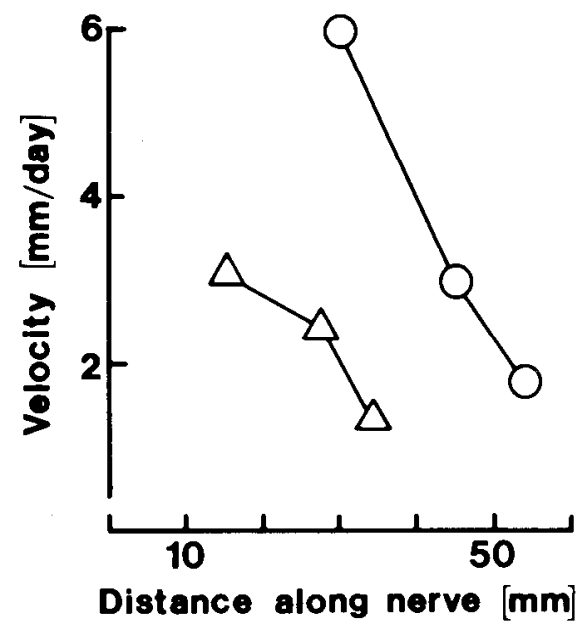

2b filament-associated radioactivity along the nerve (Griffin et al., 1984). The 145-kilodalton band was removed from the gels and dissolved in hydrogen peroxide. Levels of radioactivity were measured using liquid scintillation spectroscopy (Hoffman and Lasek, 1975) and plotted as a function of distance along the nerve.

Measurement of transport velocity. Radioactivity in the slow component of axonal transport is distributed in a well defined wave, the location of which shifts distally along the nerve with increasing time after labeling. This wave coincides spatially with the distribution of labeled neurofilament proteins along the nerve (Hoffman and Lasek, 1975). Therefore, the distribution of labeled neurofilament proteins could be measured directly (i.e., using the 145-kilodalton protein) or inferred from the distribution of slow component radioactivity along the nerve. The position of labeled proteins along the nerve (i.e., either the 145 kilodalton protein or total slow component radioactivity) was measured at each postlabeling interval using two parameters: the location of the 50th percentile of radioactivity (Hoffman et al., 1983); and the location of the leading edge of the wave. The 50th percentile value cannot be used at postlabeling intervals when only part of the wave is located in the portion of the nerve used for analysis. In addition, the 50th percentile cannot be used for proteins such as actin and tubulin which are locally retained as the slow component wave passes distally along the nerve (Griffin et al., 1984). Therefore, in order to use the 50th percentile of radioactivity in the slow component as an accurate indicator of the location of the neurofilament proteins, it was necessary to subtract, as background, radioactivity retained along the nerve after passage of the wave (i.e., the portion trailing behind the wave)

Velocities of the 50th percentile of radioactivity and the leading edge of the wave were calculated by taking the difference between the locations at successive times after labeling and dividing this distance by the length of the time interval (i.e., distance 2 - distance 1/time 2 - time 1). For example, in neurons labeled at 3 weeks of age, the leading edge of the wave was located $30 \mathrm{~mm}$ along the nerve at 5 days after labeling and $45 \mathrm{~mm}$ at 10 days; thus, the interval velocity between 5 and 10 days after labeling was $3.0 \mathrm{~mm} /$ day (i.e., $(45-30) \mathrm{mm} /(10-5)$ days).

Preparation of tissue for morphometric analysis. At $3,4,5,8,10,12$, and 18 weeks of age, animals were anesthetized with chloral hydrate $(400 \mathrm{mg} /$ $\mathrm{kg}$, i.p.) and perfused through the ascending aorta with $0.9 \%$ saline followed hy $5 \%$ glutaraldehyde in $0.1 \mathrm{M}$ phosphate buffer $(\mathrm{pH} 7.3)$. Three animals were perfused at each age except at 18 weeks where a single animal was used. After the animals were stored at $4^{\circ} \mathrm{C}$ for $18 \mathrm{hr}$, L5 ventral roots (one from each animal) wore removed and thoroughly rinsed with $0.1 \mathrm{M}$ phosphate buffer. Three-millimeter segments obtained from the proximal level of the roots (i.e., adjacent to the spinal cord) were postfixed in $2 \%$ osmium tetroxide, dehydrated in alcohol, and embedded in Epon. Al 3, 4, 8, and 12 weeks of age, segments from the distal level of the ventral root (i.e., adjacent to the dorsal root ganglion) were also processed in the same way. Transverse sections ( $1 \mu \mathrm{m}$ thick) were stained with toluidine blue for light microscopy. Thin sections (pale silver interference color), stained with lead citrate and uranyl acetate, were examined using a Hitachi model $\mathrm{H}-600$ electron microscope.

Measurement of axonal calibers. The caliber of motor axons in the L5 ventral root was measured at $3,4,5,8,12$, and 18 weeks of age. In transverse sections of these roots, four nonoverlapping fields were photographed at a magnification of $\times 250$ using a Zeiss Photomicroscope 111 and printed at a final magnification of $\times 2000$. Axonal areas were measured using either a Hewlett-Packard model 9864 digitizer interfaced with a model

Figure 2. $a$, The levels of radioactivity in the 145-kilodalton neurofilament protein are plotted as a function of distance along the sciatic nerve 5,10 , 15 , and 20 days afler labeling lurnbar motor neurons in 3-week-old animals by the intraspinal injection of $\left.{ }^{35} S\right]$ methionine. The gels used in this analysis are shown in Figure 1. Radioactivity is distributed in a well defined wave. The location of the leading edge of this wave is indicated by vertical arrows. The location of the leading edge could not be determined at 20 days after labeling because radioactive protein extended distally beyond the region of the nerve analyzed in this study. These profiles were normalized in order to facilitate their comparison. The peak levels of radioactivity in these profiles were 7612 $\mathrm{dpm}$ at 5 days, $3640 \mathrm{dpm}$ at 10 days, $1864 \mathrm{dpm}$ at 15 days, and $1860 \mathrm{dpm}$ at 20 days. $b$, Slowing of neurofilament transport along motor fibers labeled at 3 weeks of age. Velocities of the 50th percentile of radioactivity in the 145 -kilodalton neurofilament protein $(\triangle)$ and the leading edge of the wave (O) are plotted as a function of the location of the 50th percentile or the leading edge at 5, 10, and 15 days after labeling for the $0-$ to 5-, 5- to 10-, and 10- to 15-day intervals, respectively (see the text). Velocities of both the 50 th percentile and the leading edge progressively decline with increasing distance along the nerve 

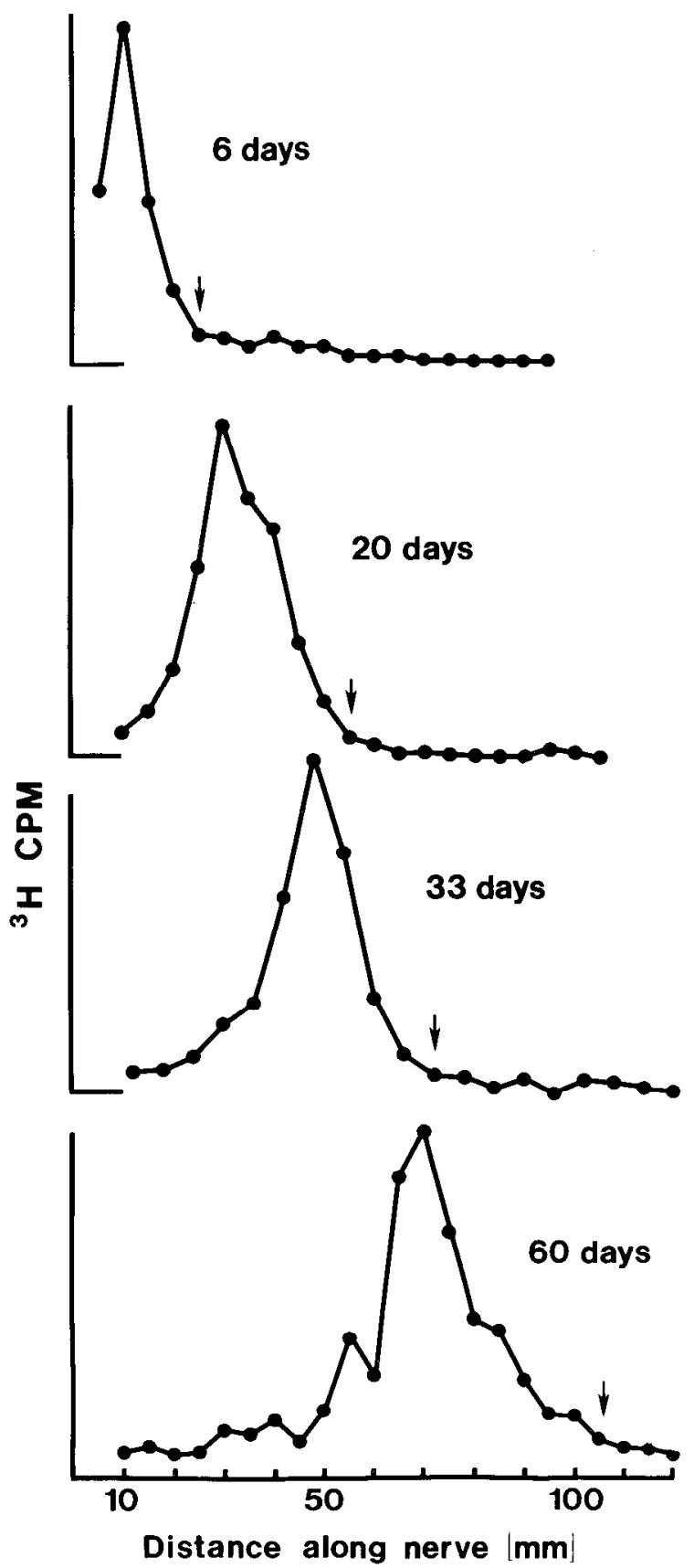

3a

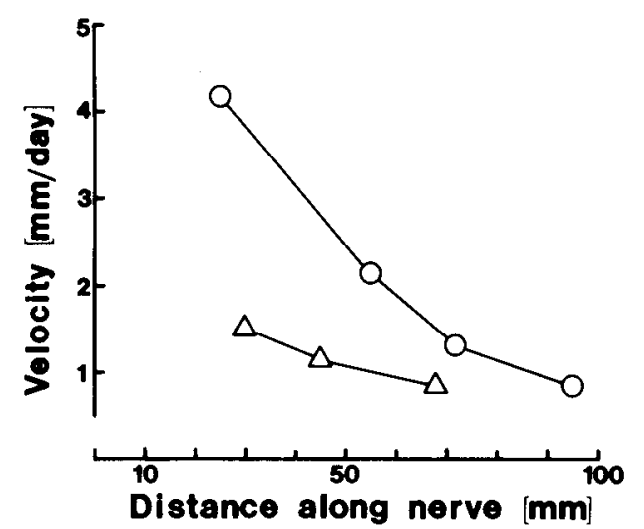

3b 9815s programmable calculator (8-, 12-, and 18-week-old animals) or a Houston $\mathrm{Hi}$-Pad digitizer interfaced with an Apple II computer and appropriate software (Bioquant, R \& M Biometrics, Nashville, TN) (3-, 4-, and 5-week-old animals). In these fields, caliber was only measured in the internodal segments of myelinated nerve fibers (i.e., sections through the nodes of Ranvier, paranodes, and Schmidt-Lanterman clefts were not included). Axonal diameters were calculated from the measured areas. At least 200 axons were analyzed in each root and three roots were analyzed in each age group (except at 18 weeks). For each root, we determined the percentage of axons in each size class; histograms were constructed by plotting the mean percentage of axons in each size class (i.e., from all of the roots in that age group) as a function of axonal diameter. In addition, we determined the mean areas of the largest $25 \%$ of the axons and the smallest $25 \%$ of the axons in each root and for each age group.

Axonal diameters were compared at the proximal and distal levels of the roots in 3-, 4-, 8-, and 12-week-old animals. Differences between the calibers of the largest axons at the proximal and distal levels were evaluated as follows. First, we found the proportion of axons at the proximal and distal levels of each root with diameters greater than the 75th percentile value for the combined proximal and distal data. Second, we used the Mantel Haenszel test to evaluate variability among these proportions at each level for the roots analyzed at a given age. If variability was not significant, data from the individual roots (i.e., for each level) were pooled. Third, the statistical significance of differences in the proportion of axons at the proximal and distal levels with diameters greater than the 75th percentile was assessed using the $\chi^{2}$ test.

Counting axonat neurofilaments. Neurofilaments were counted ir molor axons of 3-and 10-week-old animals. Thin sections of the roots were placed on Formvar-coated grids, stained with uranyl acetate and lead citrate, and examined using electron microscopy. Fields of axoplasm were photographed at a magnification of $\times 30,000$ and printed at a final magnification of $x$ 75,000 . In some cases, entire axons were photographed using overlapping fields and reconstructed using montages (Hoffman et al., 1984a). In other instances, representative fields of axoplasm were photographed for counting neurofilaments. In both cases, we measured the area of the axoplasm in which neurofilaments were counted and total axonal area. The majority of neurofilaments were sectioned transversely, making them easy to identify; neurofilaments sectioned obliquely were counted only if they could be unequivocally distinguished from other axonal constituents. The number of neurofilaments in each axon was plotted as a function of the cross-sectional area of that axon.

\section{Results}

Axonal transport of neurofilament proteins. Following the injection of $\left.{ }^{35} \mathrm{~S}\right]$ methionine into the lumbar spinal cords of 3-week-old animals, the neurofilament triplet proteins (200,145, and 68 kilodaltoris), actirı (43 kilodaltons), and tubulin (55 kilodaltons) were transported distally along motor fibers. The distributions of the neurofilament proteins coincided with one another at each postlabeling interval (Fig. 1). The distributions of the neurofilament proteins differed from those of actin and tubulin in two ways. First, at each time period, the neurofilament proteins were not transported as rapidly as the fastest moving fractions of tubulin and actin. Second, significant amounts of labeled tubulin and actin were retained in the proximal portion of the nerve after the passage of the slow component wave, whereas the neurofilament proteins showed relatively little retention (Fig. 1).

The velocity of the neurofilament proteins declined with increasing time after labeling (Fig. 1). In neurons labeled at 10 weeks of age,

Figure 3. a, Distribution of neurofilament proteins in motor neurons labeled at 12 weeks of age. The levels of radioactivity in the 145-kilodalton neurofilament protein are plotted as a function of distance along the sciatic nerve $6,20,33$, and 60 days after labeling lumbar motor neurons by the intraspinal injection of $\left[{ }^{3} \mathrm{H}\right]$ leucine and $\left[{ }^{3} \mathrm{H}\right] \mathrm{lysine}$. This radioactivity is distributed in a well defined wave; the location of the leading edge of this wave is indicated by vertical arrows. These profiles were normalized in order to facilitate their comparison. The peak levels of radioactivity in these profiles were $5686 \mathrm{dpm}$ at 6 days, $2500 \mathrm{dpm}$ at 20 days, $2105 \mathrm{dpm}$ at 33 days, and $940 \mathrm{dpm}$ at 60 days. $b$, Slowing of neurofilament transport along motor fibers labcled at 12 weeks of age. Velocities of the 50th percentile of radioactivity in the 145kilodalton neurofilament protein $(\triangle)$ and the leading edge of the wave $(O)$ are plotted as a function of distance along the nerve at the end of each interval (see Fig. 2b). Velocities of both the 50th percentile and the leading edge progressively decline with increasing distance along the nerve. 
the 50th percentile of radioactivity in the slow component wave was located at distances along the nerve of $40.1 \pm 1.4(n=4)$ and 60.4 $\pm 3.9(n=5) \mathrm{mm}$, respectively, at 33 and 60 days after labeling (see Hoffman et al., 1983, for transport profiles). The velocity between 0 and 33 days after labeling (i.e., $1.22 \pm 0.04 \mathrm{~mm} /$ day) was significantly greater ( $p<0.001$, two-sample $t$ test) than the velocity between 33 and 60 days (i.e., $0.75 \pm 0.07 \mathrm{~mm} /$ day). Thus, the velocity of the slow component wave (and the neurofilament proteins within this wave) declined with increasing time after labeling.

Reductions in velocity correlated both with age and with the position of neurofilament proteins along the nerve. Previous studies have shown that the velocity of the slow component declines with age (Droz, 1965; Lasek, 1970, Hendrickson and Cowan, 1971; Komiya, 1980; Hoffman et al., 1983). This was seen when velocity was compared in neurons labeled at 10 and 20 weeks of age. The 50 th percentile of radioactivity in the slow component wave was located $31.2 \pm 3.7 \mathrm{~mm}$ along the nerve 33 days after labeling neurons in 20-week-old animals. The velocity between 0 and 33 days after labeling was significantly greater $(p<0.001$, two-sample t test) in neurons labeled at 10 (i.e., $1.22 \pm 0.04 \mathrm{~mm} /$ day) than at 20 weeks of age (i.e., $0.95 \pm 0.11 \mathrm{~mm} /$ day).

Reductions in velocity related to position along the nerve were separated from those due to aging by comparing the velocity of the slow component wave in different regions of the nerve in animals of comparable age. At 60 and 85 days after labeling neurons in 10week-old animals (i.e., at 19 and 22 weeks of age, respectively), the 50 th percentile of radioactivity in the slow component wave was located at mean distances of $60.4 \pm 3.9(n=5)$ and $74.9 \pm 9.9$ $\mathrm{mm}(n=5)$, respectively. Thus, between 19 and 22 weeks of age, labeled proteins moved along the distal portion of the nerve (i.e., from 60.4 to $74.9 \mathrm{~mm}$ ) at a velocity of $0.58 \pm 0.19 \mathrm{~mm} /$ day. This was significantly less ( $p<0.001$, two-sample $t$ test) than the velocity (i.e., $0.95 \pm 0.11 \mathrm{~mm} /$ day between 0 and 33 days) at which proteins labeled at 20 weeks moved along the proximal portion of the nerve (i.e., from 0 to $31.2 \mathrm{~mm}$ ). Thus, in animals of comparable age, the velocity of the slow component wave (and the neurofilament proteins within this wave) was significantly greater in proximal than in distal regions of the nerve. Progressive reduction in the velocity of neurofilament proteins with increasing distance along the nerve is illustrated in neurons labeled at 3 and 12 weeks of age in Figures 2 and 3 , respectively.

Radial growth of developing nerve fibers. Myelinated axons in the L5 ventral root fall into two size classes, large and small (Figs. 4 and 5 ), which account for approximately $67 \%$ and $33 \%$ of the total fibers, respectively (Hoffman et al., 1984a). Radial growth was compared in large and small fibers, respectively, by measuring changes in the areas of the largest $25 \%$ and the smallest $25 \%$ of the axons at the proximal level of the root between 3 and 18 weeks of age (Fig. 6). In both cases, mean cross-sectional area increased with age. Linear regression analysis yielded the following relationships between age and axonal cross-sectional area: $y=2.89 x+16.30$ (correlation coefficient, 0.97 ) for the largest $25 \%$ of axons and $y=0.17 x+$ 1.91 (correlation coefficient, 0.92) for the smallest 25\%, where $y$ equals axonal area in square micrometers and $x$ equals age in weeks (Fig. 6). Thus, the rate of radial growth was approximately 17 times greater in large than in small axons (i.e., 2.89 versus $0.17 \mu \mathrm{m}^{2}$ / week, respectively).

Previous studies have shown that the caliber of mature nerve fibers is greater at internodes than at nodes of Ranvier and paranodal regions (Berthold, 1978). We found that caliber was also greater at internodes than at nodes in nerve fibers of immature (3-week-old) animals (Fig. 7).

Tapering of developing motor fibers. Axonal caliber was compared at the proximal and distal levels of the L5 ventral root in 3-, 4-, 8-, and 12-week-old animals (Fig. 8). At 3 and 4 weeks of age, the caliber of the largest fibers appeared to be greater at the proximal than at the distal level of the roots (Fig. 8). Differences between the calibers of the largest $25 \%$ of axons at the proximal and distal levels were evaluated using the $\chi^{2}$ test (see "Materials and Methods"). At 3 and 4 weeks of age, the proportion of axons with diameters greater than the 75th percentile value (for the pooled proximal and distal axons) was significantly greater proximally than distally $(p<0.001)$.
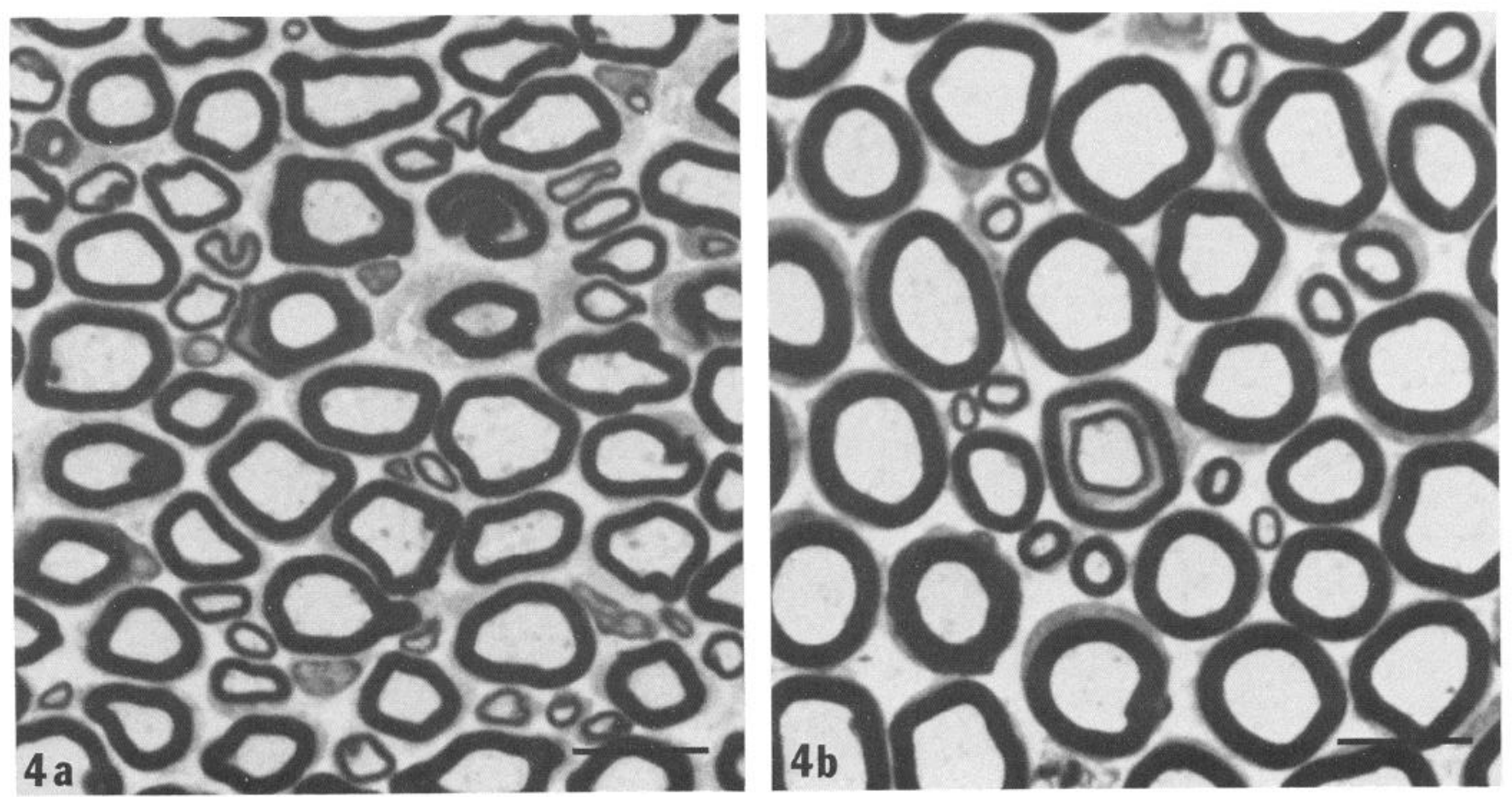

Figure 4. Radial growth of developing motor fibers. Cross-sections of axons (pale areas surrounded by darkly stained myelin sheaths) at the proximal level of the L5 ventral root in 3- (a) and 12-week-old (b) animals are compared in these light micrographs. Note the bimodal distribution of fiber sizes at both ages. Epon-embedded sections, toluidine blue stain. Magnification $\times 1600$. Bars, $10 \mu \mathrm{m}$. 
In 3-week-old animals the mean cross-sectional area of the largest $25 \%$ of axons was $20 \%$ greater proximally than distally (i.e., 25.79 $\pm 3.27 \mu \mathrm{m}^{2}$ proximally and $20.74 \pm 0.23 \mu \mathrm{m}^{2}$ distally). In contrast, axonal caliber at the proximal and distal levels was not significantly different in either 8- or 12-week-old animals. Thus, proximal-to-distal tapering of these fibers was only detected in relatively young animals.

Neurofilament content of developing motor fibers. At both 3 and 10 weeks of age, neurofilament density was equal in axons of all
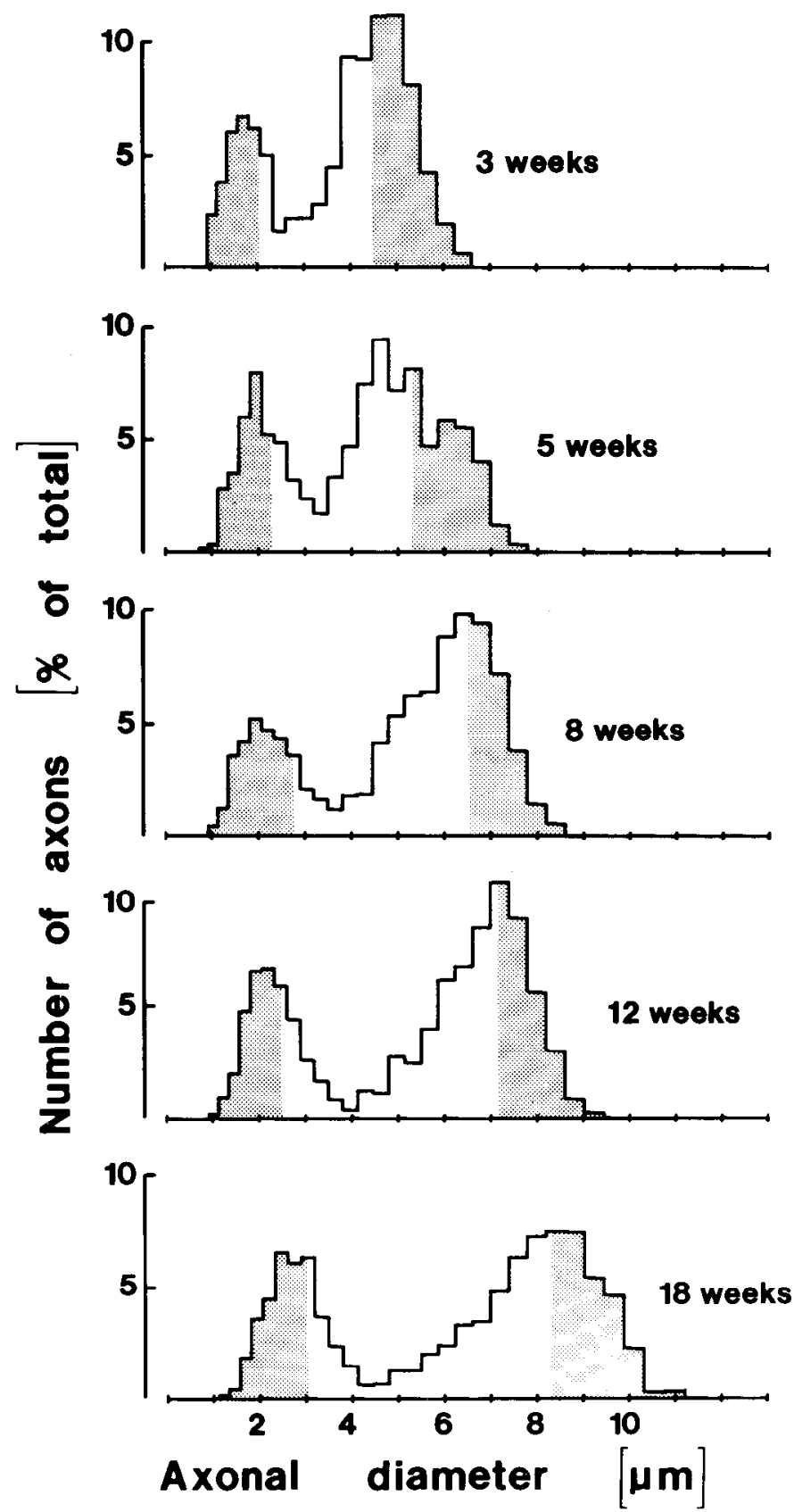

Figure 5. Kadial growth of motor axons between 3 and 18 weeks of age. Axonal caliber was measured at the proximal level of the $L 5$ ventral root. In these histograms, the mean percentage of axons in each size class is plotted as a function of axonal diameter. These axons are distributed into two well defined size classes. The largest $25 \%$ and the smallest $25 \%$ of axons, respectively, correspond to the shaded areas on the right and left sides of these histograms. In both populations axonal caliber increases with age (see Fig. 6). Three roots (from separate animals) were analyzed at each age (except for a single animal at 18 weeks). At least 200 axons were analyzed in each root.

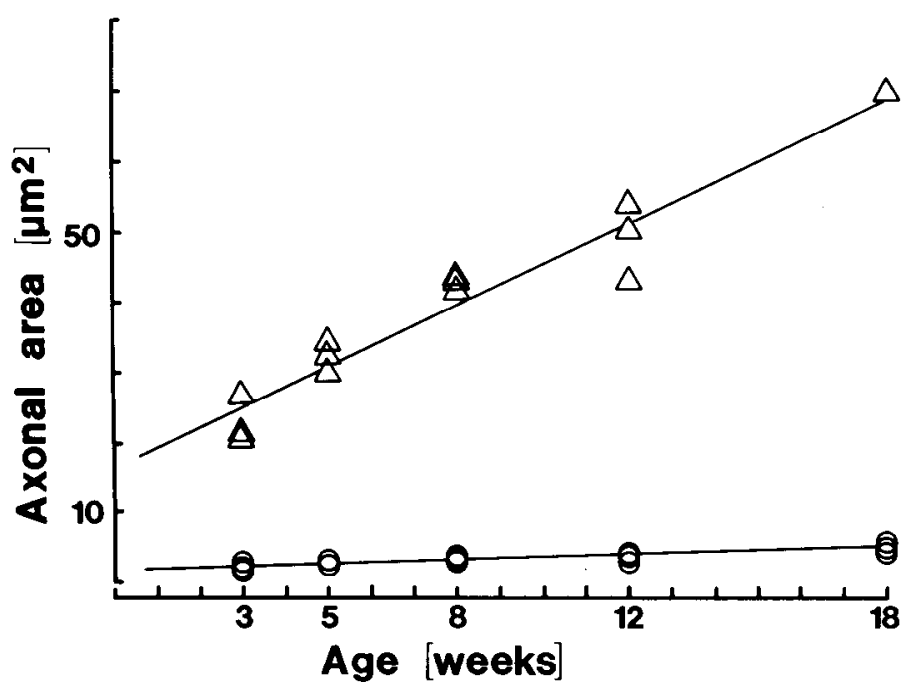

Figure 6. Increase in the cross-sectional area of motor axons between 3 and 18 weeks of age. The mean cross-sectional areas of the largest $25 \%$ $(\triangle)$ and the smallest $25 \%$ of axons at the proximal level of the $L 5$ ventral root $(O)$ are plotted as a function of age at $3,5,8,12$, and 18 weeks (see Fig. 5). In both large and small caliber axons, mcan cross scctional area appears to increase linearly with age.

sizes (Fig. 9). Since axonal cross-sectional area correlated linearly with neurofilament number, the radial growth of these fibers was associated with a proportional increase in neurofilament content. Linear regression analysis yielded the following relationship between axonal cross-sectional area and neurofilament number: $y=118 x-$ 78.8 , where $y$ equals neurofilament number and $x$ equats axonal area in square micrometers.

\section{Discussion}

Neurofilament transport in developing nerve fibers. The neurofilament proteins are synthesized exclusively in neuron cell bodies and transported along nerve fibers as part of the slow component of axonal transport (Hoffman and Lasek, 1975). In large caliber fibers (e.g., sensory and motor fibers of the sciatic nerve) these proteins account for a large fraction of the tolal radioactivily in the slow component wave (Hoffman and Lasek, 1975; Mori et al., 1979). Since the neurofilament triplet proteins are transported in a constitutive manner (Hoffman and Lasek, 1975), the distribution of any one of them (e.g., the 145-kilodalton subunit) is representative of the other two. Although several forms of the 145-kilodalton neurofilament protein appear to be transported in axons, they can be unequivocally distinguished from other labeled proteins in the slow component using one-dimensional polyacrylamide gel electrophoresis (Nixon et al., 1982). Recent evidence indicates that the neurofilament proteins undergo transport as assembled organelles (Morris and Lasek, 1982). Thus, the axonal transport of pulse-labeled neurofilament proteins (e.g., the 145-kilodalton subunit) appears to represent the somatofugal movement of neurofilaments within nerve fibers.

Labeled neurofilament proteins are distributed along the nerve in a relatively broad wave which represents the sum of radioactivity in individual nerve fibers. Previously, we reported that the width of this wave increases as it moves distally along motor fibers in developing (10-week-old) rats (Hoffman et al., 1983), and it was suggested that this increase in width reflects the presence of several populations of motor fibers within the nerve in which the velocity of these proteins differs slightly. The present observation that velocity progressively declines along the nerve is consistent with this hypothesis (i.e., that there are several populations of motor fibers). In spite of the overall decline in velocity along the nerve, differences in velocity would persist, leading to an increase in the width of the wave as it moves distally. 


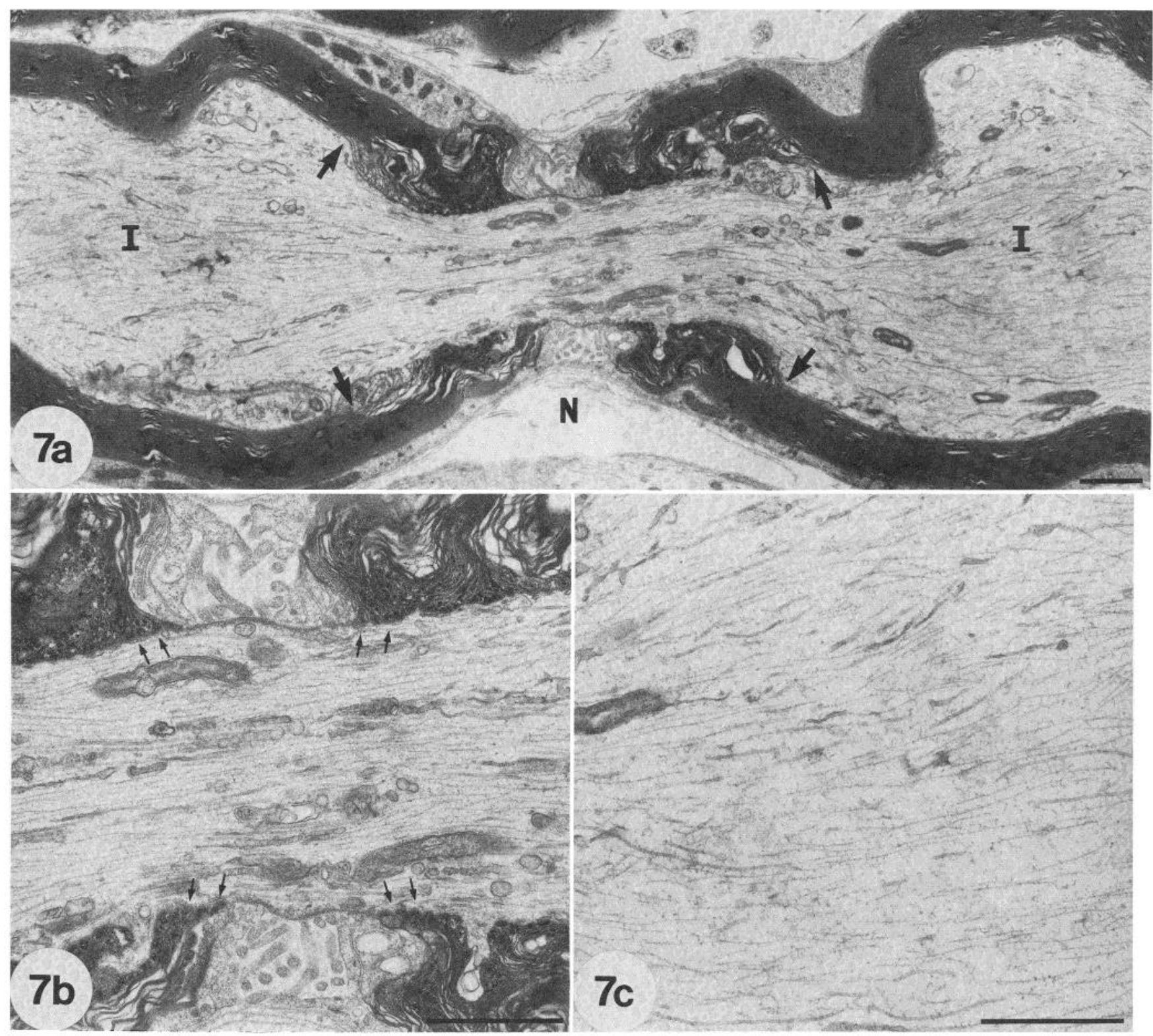

Figure 7. Longitudinal view of a constricted segment. a, Low power electron micrograph demonstrates that the caliber of the constricted segment is significantly less than that of the internodes $(I)$ in a motor fiber from a 3-week-old animal. The constricted segment, which is comprised of the node of Ranvier $(N)$ and the paranodal regions, is delineated by large arrows. $b$, Myelin attachment sites (small arrows) are present in the paranodal regions. $c$, Higher power view of the internode demonstrates that interfilament distance is greater in internodes than in nodes $(b)$. Magnification: $a, \times 9,600 ; b, \times$ 21,$000 ; c, \times 22,000$. Bars, $1 \mu \mathrm{m}$.

The role of neurofilaments in radial growth. Several lines of evidence indicate that neurofilaments are major intrinsic determinants of axonal caliber in myelinated nerve fibers (Hoffman et al., $1984 a, b)$. They are the most numerous cytoskeletal structures in the internodal segments of myelinated nerve fibers, outnumbering microtubules by more than 10:1 in large caliber fibers (Friede and Samorajski, 1970; Weiss and Mayr, 1971; Berthold, 1978). Neurofilaments and their surrounding domains occupy most of the crosssectional area in these axons (Zenker et al., 1972; Hoffman et al., 1984a), and neurofilament number is linearly correlated with axonal cross-sectional area (Friede and Samorajski, 1970; Weiss and Mayr, 1971; Berthold, 1978; Hoffman et al., 1984a). Reduction in axonal caliber in the proximal stumps of transected nerve fibers correlates with a proportionl decrease in the number of axonal neurofilaments, but not microtubules (Hoffman et al., 1984a). In the present study we found that the radial growth of developing nerve fibers correlated with a proportional increase in axonal neurofilament content. Taken together, these observations suggest that the radial growth of developing nerve fibers results directly from an increase in neurofilament content.

It should be noted that neurofilament content is not a major determinant of axonal diameter in small caliber nerve fibers (i.e., mature unmyelinated fibers and embryonic nerve fibers prior to myelination, both of which have diameters less than $1 \mu \mathrm{m}$ ) (Berthold, 1978; Sasaki-Sherrington et al., 1984). In these fibers the cytoskeleton consists largely of microtubules with relatively few neurofilaments (Peters and Vaughn, 1967; Berthold, 1978). During radial growth, the number of neurofilaments in myelinated axons increases faster than the number of microtubules; as a result, neurofilaments outnumber microtubules in mature nerve fibers (Friede and Samorajski, 1970; Berthold, 1978).

Unlike neurofilaments, microtubules do not appear to play a 

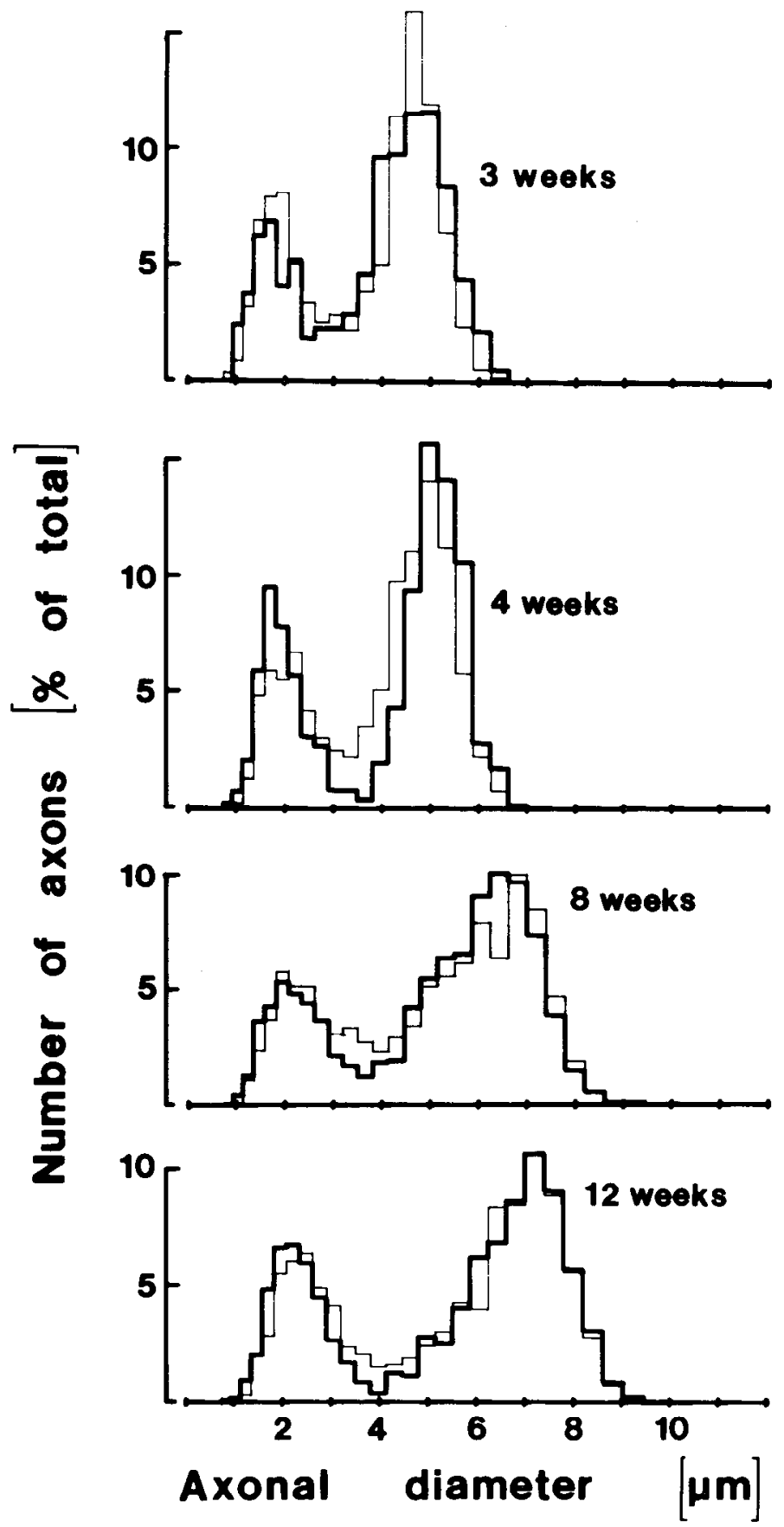

Figure 8. Calibers of motor axons at proximal and distal levels of the $L 5$ ventral root. Axonal caliber was measured at the proximal (bold lines) and distal levels (thin lines) of three separate roots (from different animals) at 3 , 4,8 , and 12 weeks of age. The mean percentage of axons in each size class is plotted as a function of axonal diameter. At least 200 axons were analyzed in each root.

prominent role in the radial growth of large-caliber nerve fibers. In motor fibers of rat $L 5$ ventral root, microtubule number is maximal in axons with cross-sectional areas of approximately $30 \mu \mathrm{m}^{2}$ and remains nearly constant with further increases in area (Hoffman et al., 1984a). Therefore, the radial growth of axons with cross-sectional areas greater than $30 \mu \mathrm{m}^{2}$ (i.e., the largest $25 \%$ of axons in rats 8 or more weeks of age) correlates with little, it any, change in the number of microtubules.

Regional heterogeneity of axoplasm. In myelinated nerve fibers there are substantial differences in the organization of the cyto-

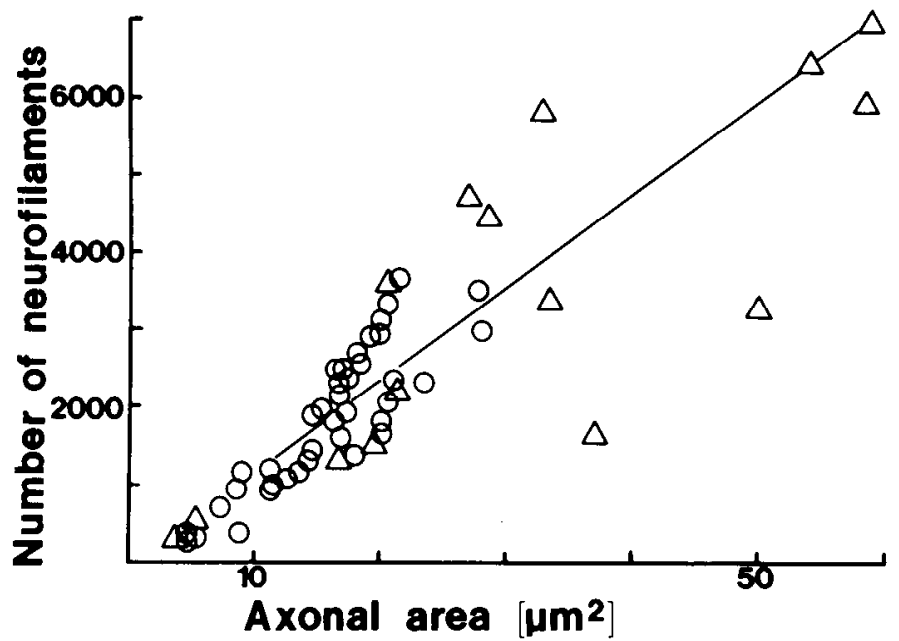

Figure 9. Increase in neurofilament number in motor axons undergoing radial growth. The number of neurofilaments per axon is plotted as a function of axonal cross-sectional area in the motor fibers of 3- $(\mathrm{O})$ and 10-week-old $(\triangle)$ animals. In both cases, neurofilament number increases linearly with axonal cross-sectional area.

skeleton at the internodes and the constricted segments, i.e., nodes of Ranvier and paranodal regions (Berthold, 1978). The internodal segments of individual fibers (i.e., the regions between successive nodes) are equal in length; in large fibers of the rat $L 5$ ventral root the internodes are approximately $500 \mu \mathrm{m}$ long at 3 weeks of age, increasing to $1500 \mu \mathrm{m}$ by 8 weeks (J. W. Griffin, P. N. Hoffman, K. A. Fahnestock, and M. P. Besaquen, submitted for publication). In mature nerve fibers, axonal cross-sectional area at the nodes may be only $10 \%$ that of the internodes (Berthold, 1978). Using skip serial sections of individual nerve fibers, Berthold (1978) found that neurofilament number is substantially greater at internodes than at nodes. In contrast, the number of microtubules is comparable at nodes and internodes (Berthold, 1978). Therefore, both the density of microtubules and the ratio of microtubules to neurofilaments are significantly greater at nodes than at internodes.

Mechanisms of radial growth. Differences in composition of the cytoskeleton at nodes and internodes suggest that there must be reorganization of the cytoskeleton as it is transported through successive nodes. Hoffman and Lasek (1975) viewed the the cytoskeleton as a coherent network of microtubules and neurofilaments in which the relative positions remain fixed during transport along the nerve. This model does not account for differences in the composition of the cytoskeleton at nodes and internodes. We will now consider how progressive reduction in the velocity of neurofilament transport along the nerve could provide a mechanism for synchronous radial growth and also account for the observed differences in composition of the cytoskeleton at nodes and internodes.

The neurofilament content of neve fibers appears to be regulated through the axonal transport of neurofilaments (Hoffman et al., $1984 a, b)$. Local turnover and assembly do not normally appear to play major roles in determining axonal neurofilament content. The neurofilament proteins appear to be transported exclusively in their polymeric (filamentous) form (Morris and Lasek, 1982), precluding local assembly. In addition, there is little, if any, turnover of neurofilaments along the axon (Lasek and Black, 1977); turnover appears to be confined to the axon terminals (Lasek and Hoffman, 1976; Roots, 1983). Therefore, in order for local neurofilament content to increase during radial growth, the quantity of neurofilaments entering a region per unit time (via axonal transport) must exceed the quantity leaving.

Direct evidence relating the axonal transport of neurofilaments to the control of caliber comes from a recent study which examined 
the spatial and temporal evolution of reduction in caliber in the proximal stumps of transected motor fibers (Hoffman et al., 1984a). This wave of axonal atrophy, which correlates with a proportional decrease in neurofilarnent number, begiris proximally and spreads along fibers in a proximal-to-distal direction at a rate equal to the velocity of neurofilament transport (Hoffman et al., 1984a). The timing and severity of reductions in axonal caliber and neurofilament content correlate with a decrease in the relative amount of neurofilament protein transported into these nerve fibers (Hoffman and Lasek, 1980; Hoffman et al., 1985). After axotomy, reduced quantities of neurofilaments enter the proximal axon from the cell body; at the same time, normal quantities of neurofilaments leave this region (via transport to more distal regions of the nerve). Thus, when the quantity of neurofilaments leaving the proximal axon exceeds the quantity entering from the cell body, neurofilament content declines, resulting in a secondary decrease in axonal caliber. Presumably, the converse is true for axons undergoing radial growth; i.e., axonal caliber and neurofilament content increase when the quantity of neurofilaments entering a region exceeds the quantity leaving.

One mechanism for increasing the quantity of neurofilaments entering a region is through increments in neurofilament synthesis Lasek et al., (1983) have proposed that neurofilament synthesis increases during the radial growth of developing nerve fibers. Thus, by increasing the quantity of neurofilaments entering the axon, the quantity entering a given region could exceed the quantity leaving, allowing local neurofilament content to increase. Accordingly, neurofilament number would be slightly greater proximally than distally (i.e., since neurofilaments enter the axon from the cell body). This could account for the proximal-to-distal tapering of axons observed in 3- and 4-week-old animals.

A second mechanism contributing to the radial growth of developing axons is the progressive reduction in the velocity of neurofilament transport along the nerve. As a consequence of this slowing, the velocity at which neurofilaments enter each internodal segment would be greater than the velocity at which they leave. This would allow for simultaneous increase in the neurofilament content of every internode and would contribute to the synchronous radial growth of developing nerve fibers. If this reduction in velocity occurs exclusively within the internodes (and not at the nodes), then neurofilament content would increase within the internodes, and, at the same time, remain constant at the nodes. This would account for differences in the composition of the cytoskeleton in these regions.

In at least one situation, reduction in velocity appears to be directly responsible for substantial increments in axonal caliber and neurofilament content. After systemic intoxication with the neurotoxin $\beta, \beta^{\prime}$. iminodipropionitrile, marked reduction in neurofilament velocity leads to dramatic increases in caliber and neurofilament content in the proximal axon. In this model of cytoskeleton pathology, axonal enlargement reflects a mismatch between the delivery of newly synthesized neurofilaments from nerve cell bodies (to the proximal axon) and the ability to translocate these organelles along the axon in a proximal-to-distal direction (Griffin et al., 1978; Griffin et al., 1983b). Thus, alterations in velocity can profoundly influence the neurofilament content of axons.

At present we can only speculate as to the factors responsible for the slowing of neurofilament transport in developing nerve fibers. One possibility is that neurofilament velocity is regulated by growth of the axolemma or the subaxolemmal cytoskeieton, with neurofilaments arriving by axonal transport and then passively filling the space in order to maintain a constant density. However, we think it more likely that a regulatory process directly affects neurofilament volocity, with cosequent adjustment of the axolemma (i.e., in response to changes in axonal caliber). It is likely that the mechanism of neurofilament transport involves interactions between neurofilaments and other axonal structures such as microtubules or the axolemma (Hoffman and Lasek, 1975; Griffin et al., 1983a). In theory, it is possible that post-translational modification of neurofilament proteins as they are transported within the axon could lead to alterations in these interactions and thereby change neurofilament velocity. Once modified, the neurofilaments would continue to move at their new (reduced) rate until further modification led to additional reductions in velocity. This hypothesis is consistent with the observation that there are progressive alterations in the 145-kilodalton neurofilament protein (resulting in changes in its electrophoretic mobility) as it is transported distally in axons of mouse optic nerve (Nixon et al., 1982).

One potential way in which the neurofilament proteins could be modified is through changes in their level of phosphorylation. These proteins, particularly the 200- and 145-kilodalton subunits, are highly phosphorylated (Pant et al., 1978; Julien and Mushynski, 1982). In fact, recent evidence suggests that the degree of phosphorylation of the 200-kilodalton protein differs in specific regions of the neuron (Sternberger and Sternberger, 1984). We propose that post-translational modifications of the neurofilament proteins (e.g., changes in the level of phosphorylation) occur as they are transported along axons, lcading to a progressive decline in neurofilament velocity. This molecular model provides an explanation for the progressive decline in neurofilament velocity which we believe contributes to the radial growth of axons during development.

\section{References}

Aguayo, A. J., G. M. Bray, C. S. Perkins, and I. D. Duncan (1979) Axonsheath cell interactions in peripheral and central nervous system transplants. In Society for Neuroscience Symposia. Vol. 4: Aspects of Developmental Neurobiology, pp. 361-383, Society for Neuroscience, Bethesda, MD.

Berthold, C. H. (1978) Morphology of normal peripheral axons. In Physiology and Pathobiology of Axons, S. G. Waxman, ed., pp. 3-63, Raven Press, New York.

Droz, B. (1965) Fate of newly synthesized proteins in neurons. In The use of Radioautography in Investigating Protein Synthesis, C. P. Leblond and K. B. Warren, eds., pp. 159-174, Academic Press, Inc., New York.

Friede, R. L., and A. J. Martinez (1970) Analysis of the process of sheath expansion in swollen nerve fibers. Brain Res. 19: 165-182.

Friede, R. L., and T. Samorajski (1970) Axon caliber related to neurofilaments and microtubules in sciatic nerve fibers of rats and mice. Anat. Rec. 167. 379-387.

Griffin, J. W., P. N. Hoffman, A. W. Clark, P. T. Carroll, and D. L. Price (1978) Slow axonal transport of neurofilament proteins: Impairment by $\beta, \beta^{\prime}$ iminodipropionitrile administration. Science 202: 633-635.

Griffin, J. W. K. E. Fahnestock, D. L. Price, and P. N. Hoffman (1983a) Microtubule-neurofilament segregation produced by iminodipropionitrile administration: Evidence for the association of fast axonal transport with microtubules. J. Neurosci. 3: 557-566.

Griffin, J. W., D. L. Price, and P. N. Hoffman (1983b) Neurotoxic probes of the axonal cytoskeleton. Trends Neurosci. 6: 490-495.

Griffin, J. W. D. C. Anthony, K. E. Fahnestock, P. N. Hoffman, and D. G. Graham (1984) 3,4-Dimethyl-2,5-hexanedione impairs the axonal transport of neurofilament proteins. J. Neurosci. 4: 1516-1526.

Hendrickson, A. E., and W. M. Cowan (1971) Changes in the rate of axoplasmic transport during postnatal develoment of the rabbit's. optic nerve and tract. Exp. Neurol. 30: 403-422.

Hoffman, P. N., and R. J. Lasek (1975) The slow component of axona transport: Identification of the major structural polypeptides of the axon and their generality among mammalian neurons. J. Cell Biol. 66: 351-366.

Hoffman, P. N., and R. J. Lasek (1980) Axonal transport of the cytoskeleton in regenerating motor neurons: Constancy and change. Brair Res. 202. 317-333.

Hoffman, P. N., R. J. Lasek, J. W. Griffin, and D. L. Price (1983) Slowing of the axonal transport of neurofilament protein during development. $\mathrm{J}$. Neurosci. 3: 1694-1700.

Hoffman, P. N., J. W. Griffin, and D. L. Price (1984a) Control of axonal caliber by neurofilament transport. J. Cell Biol. 99: 705-714.

Hoffman, P. N., J. W. Griffin, and D. L. Price (1984b) Neurofilament transport in axonal regeneration: Implications for the control of axonal caliber. In Advances in Neurochemistry, Axonal Transport in Growth and Regeneration, J. Elam and P. Cancalon, eds., pp. 243-260, Plenum Publishing Corp., New York. 
Hoffman, P. N., G. W. Thompson, J. W. Griffin, and D. L. Price (1985) Changes in neurofilament transport coincide temporally with alterations in the caliber of axons in regenerating motor fibers. J. Cell Biol., in press.

Hursh, J. B. (1939) Conduction velocity and diameter of nerve fibers. Am. J. Physiol. 127: 131-139.

Julien, J. -P., and W. E. Mushynski (1982) Multiple phosphorylation sites in mammalian neurofilament polypeptides. J. Biol. Chem. 257: 10467-10470

Komiya, Y. (1980) Slowing with age of the rate of slow axonal flow in bifurcating axons of rat dorsal root ganglion cells. Brain Res. 183: 477480.

Lasek, R. J. (1970) Axonal transport of proteins in dorsal root ganglion cells of the growing cat: A comparison of growing and mature neurons. Brain Res. 20: 121-126.

Lasek, R. J., and M. M. Black (1977) How do axons stop growing? Some clues from the metabolism of the proteins in the slow component of axonal transport. In Metabolism, Regulation and Special Functions of Protein Synthesis in the Brain, S. Roberts, A. Lajtha, and W. H. Gispen, eds., pp. 161-169. Elsevier/North Holland Biomedical Press, New York.

Lasek, R. J., and P. N. Hoffman (1976) The neuronal cytoskeleton, axonal transport and axonal growth. Cold Spring Harbor Conf. Cell Proliferation 3: $1021-1049$

Lasek, R. J., M. M. Oblinger, and P. F. Drake (1983) The molecular biology of neuronal geometry: The expression of neurofilament genes influences axonal diameter. Cold Spring Harbor Symp. Quant. Biol. 18: 731-714.

Minwegen, P., and R. L. Friede (1984) Conduction velocity varies with osmotically induced changes of the area of the axon's profile. Brain Res. 297: $105-113$.

Mori, H., Y. Komiya, and M. Kurokawa (1979) Slowly migrating axonal polypeptides. Inequalities in their rate and amount of transport between two branches of bifurcating axons. J. Cell Biol. 82: 174-184.

Morris, J. R., and R. J. Lasek (1982) Stable polymers of the axonal cytoskeleton: The axoplasmic ghost. J. Cell Biol. 92: 192-198.
Nixon, R. A., B. A. Brown, and C. A. Marotta (1982) Posttranslational modification of a neurofilament protein during axonal transport: Implications for regional specialization of axons. J. Cell Biol. 94: 150-158.

Pant, H. C., G. Shecket, H. Gainer, and R. J. Lasek (1978) Neurofilament protein is phosphorylated in squid giant axon. J. Cell Biol. 78: R23-27.

Peters, A., and J. E. Vaughn (1967) Microtubules and filaments in the axons and astrocytes of early postnatal rat optic nerves. J. Cell Biol. 32: 113119

Raine, C. S., H. Wisniewski, and J. Prineas (1969) An ultrastructural study of expcrimental demyclination and remyclination. II. Chronic experimental allergic encephalomyelitis in the peripheral nervous system. Lab. Invest. 21: 316-327.

Roots, B. I. (1983) Neurofilament accurnulation induced in synapses by leupeptin. Science 221: 971-972.

Sasaki-Sherrington, S. E., J. R. Jacobs, and J. K. Stevens (1984) Intracellular control of axial shape in non-uniform neurites: A serial electron microscopic analysis of organelles and microtubules in Al and All retinal amacrine cells. J. Cell Biol. 98: 1279-1290.

Sternberger, L. A., and N. H. Sternberger (1984) Monoclonal antineurofilaments probe phosphorylation in situ. Trans. Am. Soc. Neurochem. 15: 225

Stuart, D. G., and R. M. Enoka (1983) Motoneurons, motor units, and the size principle. In The Clinical Neurosciences. Vol. 5: Neurobiology, R. N. Rosenberg, ed., pp. V:471-V:517, Churchill Livingstone, New York.

Weinberg, H. J., and P. S. Spencer (1975) Studies on the control of myelinogenesis. I. Myelination of regenerating axons after entry into a foreign unmyelinated nerve. J. Neurocytol. 4: 395-418.

Neiss, P. A., and R. Mayr (1971) Organelles in neuroplasmic ("axonal") flow: Neurofilaments. Proc. Natl. Acad. Sci. U. S. A. 68:846-850.

Zenker, W., R. Mayr, and H. Gruber (1972) Axoplasmic organelles: Quantttative differences between ventral and dorsal root fibers of the rat. Experientia 29: 77-78. 